\title{
Structure of plasmas generated by the interaction between metallic ions and neutral gas particles in a low-pressure arc
}

H. Kelly, A. Lepone, and F. Minotti

Citation: Journal of Applied Physics 87, 8316 (2000); doi: 10.1063/1.373543

View online: https://doi.org/10.1063/1.373543

View Table of Contents: http://aip.scitation.org/toc/jap/87/12

Published by the American Institute of Physics

\section{Articles you may be interested in}

Electrical Properties of Zirconium Ditelluride Single Crystals

AIP Conference Proceedings 1349, 1085 (2011); 10.1063/1.3606239

Influence of gas pressure and cathode composition on ion energy distributions in filtered cathodic vacuum arcs Journal of Applied Physics 83, 2965 (1998); 10.1063/1.367052

Electronic structure of $\mathrm{ZrX}_{2}(\mathrm{X}=\mathrm{Se}, \mathrm{Te})$

The Journal of Chemical Physics 148, 124707 (2018); 10.1063/1.5019316

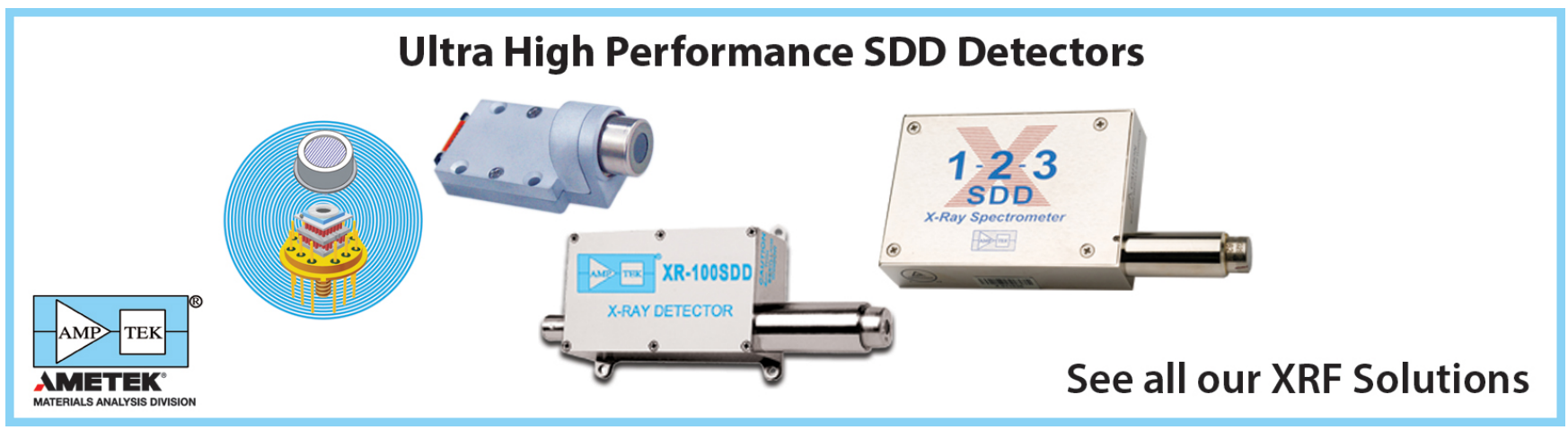




\title{
Structure of plasmas generated by the interaction between metallic ions and neutral gas particles in a low-pressure arc
}

\author{
H. Kelly, ${ }^{a), b)}$ A. Lepone, and F. Minottia) \\ Instituto de Física del Plasma (CONICET), Departamento de Física, Facultad de Ciencias Exactas, \\ y Naturales (UBA), Ciudad Universitaria Pab. I, (1428) Buenos Aires, Argentina
}

(Received 7 September 1999; accepted for publication 6 March 2000)

\begin{abstract}
A numerical solution for the metallic-plasma-neutral-gas structure generated in a low-pressure arc is presented. The equations correspond to a spherically symmetric fluid-like steady model, valid for the outer region of the arc, and describe the ion slowing down by elastic scattering with the neutral particles. Technically, the obtention of the profiles of different magnitudes is complicated due to the existence of a critical point in the steady-state system of equations. The proposed approach to overcome this difficulty is to solve instead a pseudotransient system of equations which rapidly and efficiently relax to the stationary state. By employing this numerical method of second-order accuracy in space, the plasma and neutral gas density, the electron and ion drift velocities, the electron and neutral temperatures, and the electrostatic potential profiles are obtained from the border of the arc channel up to the discharge chamber wall. It is found that the value of the neutral gas filling pressure strongly influences the plasma density and plasma potential distributions. An important result is that metallic ions emitted from the arc channel deliver their kinetic energy to the filling gas in a gradual manner, up to a pressure-dependent point beyond which they move to the walls sustained against collisions with the gas by a self-consistent electric field. Near the mentioned point, the metallic ion density presents a peculiar behavior, showing an increase that is more pronounced at high pressures; a pattern also evident in the electrostatic potential. (C) 2000 American Institute of Physics. [S0021-8979(00)07711-2]
\end{abstract}

\section{INTRODUCTION}

Low-pressure arcs operating in the cathode-spot mode are attractive because of their application to the deposition of thin films. ${ }^{1,2}$ In these devices, a high current electrical discharge is generated between conducting electrodes immersed in a vacuum (or low pressure) chamber, the current being conducted by a plasma consisting of ionized vapor of the cathode material. The emission of electrons and vapor comes from minute and mobile sites on the cathode surface, known as cathode spots. The electrons ionize the metallic vapor in the vicinities of the spot, thus producing metallic ions with different charge states which are accelerated to considerable kinetic energies (in the range of $10-100 \mathrm{eV}$, depending on the cathode material) by either the electron drag or by the presence of an electrostatic potential hump, and carrying in vacuum approximately $8 \%-10 \%$ of the total arc current. ${ }^{3,4}$ If a reactive gas such as nitrogen or oxygen is also present in the discharge chamber, the metallic ions combine chemically with the gas particles, so that metal oxide and nitride films can be produced. A typical example of cathodic vacuum arc reactive coating is the production of TiN films using a $\mathrm{Ti}$ cathode in a low-pressure $\mathrm{N}_{2}$ ambient.

It is worth noting that little is known about the metallicplasma-neutral-gas structure which is formed in the presence of a background gas. It is clear that the presence of the gas produces an attenuation of the ion current with respect to that

\footnotetext{
${ }^{a)}$ Member of the CONICET.

${ }^{b)}$ Electronic mail: kelly@tinfip.lfp.uba.ar
}

measured under vacuum conditions. The pioneering work in this respect is that of Kimblin, ${ }^{5}$ where the ionic current attenuation was determined at different distances from the arc and for different kinds of gases and pressures, using an ion collector which almost completely enclosed the arc. The magnitude of such attenuation depends on the gas type, gas pressure, and on the distance between the ion collecting probe and the electrodes.

Usual neutral-gas pressures employed with continuous arcs are in the range of $0.01-0.1 \mathrm{mbar}^{6},{ }^{6}$ but in some works, pressures in the range of 1-10 mbar are used, either with the purpose of studying the attenuation of the metallic ion flux through interactions with the neutral gas, ${ }^{5,7-9}$ or because in this high-pressure range, good reactive coatings are produced in the pulsed high-current experiments..$^{10,11}$

The study of the outer region of the discharge when the arc is operated with neutral gas filling is important because in this region, the ions lose their kinetic energy by elastic collisions with the neutral particles and some of them are recombined through inelastic processes in the neutral gas. Only a few theoretical studies have been presented on this subject, and they were concerned with the small-gap facedelectrodes geometrical configuration quoted above. Meunier and Drouet ${ }^{12}$ studied the presence of a luminous plasma "ball" whose radius $R$ was found to be dependent on the neutral gas pressure $(p)$ and $I$. This radius was interpreted as the position at which the ions suddenly delivered its kinetic energy to the gas by elastic collisions. Later on, Meunier ${ }^{13}$ proposed a snow-plow fluid model to study the transient expansion of the plasma jet. Boxman and Goldsmith ${ }^{14}$ pre- 
sented a zero-order model for the plasma-jet-background-gas interaction, by equating the plasma jet momentum flux with the background pressure. In all of these studies, the radius $R$ was thought to represent an impermeable barrier (shock wave) separating two gas-like volumes, the metallic plasma and the neutral gas. More recently, Kelly et al. ${ }^{15}$ presented a steady fluid model with spherical symmetry to describe the elastic interaction between metallic plasma and neutral gas in the outer region of the arc channel, taking into account the local balances of momentum and energy, and the heating and ensuing expansion of the surrounding gas. The set of equations included a charge neutrality equation, ion continuity and momentum, electron momentum and energy, and neutral momentum and energy equations. As the kinetic ion energy is much larger than their thermal energy, it was not necessary to consider the ion energy equation (when the ions were slowed down to kinetic energies of the order of the neutral temperature, both species were considered to be in thermal equilibrium). The model gave the radial profiles of the ion density and velocity, the electron temperature, the plasma electrostatic potential and the neutral density and temperature for given values of these quantities at the border of the arc channel. Besides, it was necessary to assign the values of the temperature gradients at this border. It was found that, instead of the presence of a shock wave, the ions gradually delivered their kinetic energy into a strongly heated and rarefied gas, up to a certain radial position $\left(r^{*}\right)$ were the ion energy was essentially thermal. Also, the ion range was found to be larger than the visible plasma radius ${ }^{12}$ or the theoretical predictions based on the balance of the plasmaneutral gas pressure at a sharp boundary. ${ }^{13,14}$

The model of Ref. 15 suffered from certain drawbacks: in the first place, it was not possible to obtain the plasma quantities for $r>r^{*}$ (although the neutral gas density and temperature were obtained up to the chamber wall position). This was due to the presence of a critical point in the equations of the model, which arises when the ion velocity is small enough and, as a consequence of the ion continuity equation, the ion density results quite large (in the next section, more details on this singularity will be given). In the second place, it was assumed in Ref. 15 that, in the absence of a significant metallic-ion recombination, the electron drift velocity should be equal to zero. Although this assumption looks reasonable since it was experimentally found that metallic shields biased to the ground cathode potential always collected a pure ion current, ${ }^{6-9}$ it would be desirable that the condition of zero electron drift velocity in the vicinities of the chamber wall can be obtained consistently from the model results. In the third place, the value of the radial electron temperature derivative at the border of the arc channel was taken somewhat arbitrarily as the same value corresponding to a vacuum arc (without gas filling) ${ }^{16}$ Although it was found in Ref. 15 that the electron temperature behavior was quite uncoupled from the ion dynamics, it is clear that any calculation (or estimation) of several inelastic processes between electrons and neutral gas particles requires a proper electron temperature profile. Finally, in Ref. 15 the neutral density at the border of the arc channel was selected so as to reach the desired value of the filling pressure at the chamber

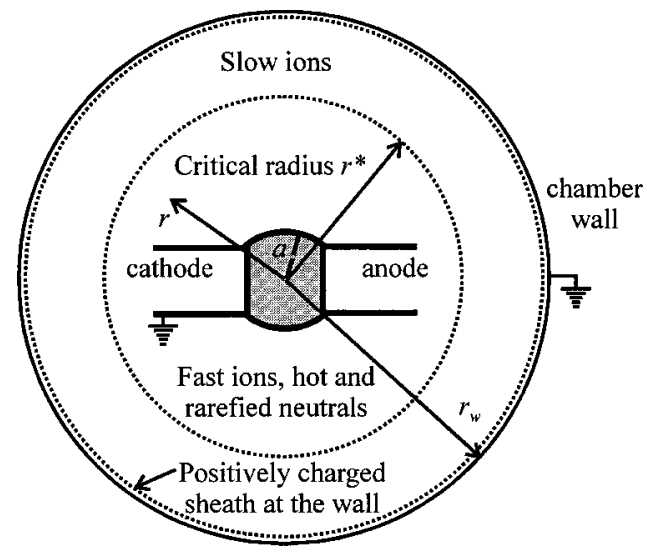

FIG. 1. Geometry employed to develop the model and main characteristics of the plasma-neutral gas structure.

wall radius $\left(r_{w}\right)$. However, it seems more reasonable to require the conservation of the neutral particle number (that is, the total number of neutral particles which, before the arc running, filled homogeneously the discharge chamber, must be equal to that obtained for the steady state neutral density structure).

Taking into account the above-mentioned limitations of the model of Ref. 15, in this work, we present an extension of that model, which allows us to obtain the plasma structure in the whole domain $a<r<r_{w}$ ( $a$ being the arc channel radius). Besides, the old boundary condition at the arc channel on the electron temperature gradient is replaced by a condition on this derivative, but at the chamber wall position. It must be advanced that this extension requires a complete change in the numerical integration scheme. While in Ref. 15 , a shooting-type method with a standard Runge-Kutta fourth-fifth order was employed, in this work, we use a relaxation method of second-order accuracy in space, to avoid the intrinsic difficulties of a shooting method with critical points.

\section{STATEMENT OF THE MODEL, ANALYSIS OF THE CRITICAL POINT, AND NUMERICAL SCHEME}

The equations to be presented in what follows are applied to the so-called multicathode spot vacuum arc, ${ }^{17}$ because arcs operating in this high-current mode have a welldeveloped interelectrode plasma region. The geometry employed in the model and the main characteristics of the plasma-gas structure are outlined in Fig. 1. The device consists in two electrodes facing each other and enclosed in a discharge chamber whose walls and cathode are grounded. The discharge is represented by an spherical "black box" of radius $a$ at a potential $V_{A}\left(V_{A}\right.$ is the anode potential, which is expected to be close to the plasma potential in the arc) acting as an ion source for the outer region, which is filled with neutral plasma and with neutral gas particles of mass $m_{n}$ at temperature $T_{n}(r)$ and density $N_{n}(r)$. The plasma electrons have temperature $T_{e}(r)$ and density $n_{e}(r)$; the plasma ions have mass $m_{i}$, charge $z_{r}$, kinetic energy $E_{i}(r)=m_{i} v_{i}^{2} / 2$ and density $n_{i}(r)$. Due to the similarity of their masses, metallic ions and neutrals are considered to be in thermal equilibrium. 
The simplifying assumptions of the model are the following: (i) Assumption of a neutral plasma, justified by the smallness of the Debye length $\left(\lambda_{D}\right)$, which for the plasma conditions considered $\quad\left(T_{e} \sim 1 \mathrm{eV} ; \quad n_{e} \sim 10^{12} \mathrm{~cm}^{-3}\right) \quad$ is $\quad \lambda_{D} \sim 5$ $\times 10^{-6} \mathrm{~cm}$, well below any other characteristic length. (ii) Spherical symmetry, supported by the experimental observation of remarkably spherical distributions of luminosity in the space around the electrodes, found in experiments performed at relatively high background gas pressures. ${ }^{12}$ (iii) Neglect of the electron inertia in the electron momentum equation (iv) Although the cathode emission involves several ions differing in their kinetic energy and charge, in order to determine the plasma-gas structure, a single ion species is assumed, averaged in charge and energy. In addition, as a first approximation consistent with the assumed spherical symmetry, the angular dependence of the ion flux ${ }^{18}$ is neglected (v) No metal vapor is considered in the region outside the electrodes.

With the above quoted considerations, the equations that determine the plasma structure in the outer region of the discharge are as follow.

The stationary ion flow continuity equation:

$$
d / d r\left(r^{2} n_{i} v_{i}\right)=-\left(1 / z_{r}\right) \sigma_{\text {loss }} r^{2} N_{n} n_{i} v_{i},
$$

where $\sigma_{\text {loss }}$ is the cross section for metallic-ion losses due to recombination.

The corresponding equation for the electron flow:

$$
d / d r\left(r^{2} n_{e} v_{e}\right)=-\sigma_{\text {loss }} r^{2} N_{n} n_{i} v_{i} .
$$

The charge neutrality condition:

$$
n_{e}=z_{r} n_{i}
$$

The conservation of ion momentum:

$$
\begin{array}{r}
m_{i} v_{i} d v_{i} / d r=-1 / n_{i} d / d r\left(n_{i} T_{n}\right)-z_{r} e d \varphi / d r+R_{i e} \\
-1 / 2 m_{i} v_{i}^{2} N_{n}\left[2 m_{n} /\left(m_{i}+m_{n}\right)\right] \sigma_{i n},
\end{array}
$$

where $\varphi$ is the plasma electrostatic potential, $R_{i e}=-z_{r} R_{e i}$ $=-(1 / \sigma) e^{2} z_{r}^{2} n_{i} v_{i}+\beta d T_{e} / d r$ is the friction force of electrons on ions ( $\sigma$ is the plasma conductivity, $\beta$ is Braginskii's thermoelectric coefficient as used in Ref. 19), $\sigma_{i n}$ is the cross section for elastic scattering between ions and neutrals. $\sigma_{i n}$ is expressed as a function of the ion kinetic energy as ${ }^{20}$

$$
\sigma_{i n}=\sigma_{0}\left[E_{0} /\left(E_{i}(r)+U\right)\right]^{1 / 2},
$$

where $\sigma_{0}=2 \times 10^{-15} \mathrm{~cm}^{2} . E_{0}=10 \mathrm{eV}$ and $U=8 \mathrm{eV}$ are constants chosen to fit the data in Ref. 20.

The conservation of electron momentum:

$$
\begin{aligned}
\left(1 / n_{e}\right) d / d r\left(n_{e} T_{e}\right)= & e d \varphi / d r+R_{e i} \\
& +m_{e} v_{e} N_{n}\left(T_{e} / m_{e}\right)^{1 / 2} \sigma_{e n} .
\end{aligned}
$$

$\sigma_{e n}$ is the cross section for elastic scattering between electrons and neutrals, taken equal to $2 \times 10^{-15} \mathrm{~cm}^{2}{ }^{21}$

The conservation of neutral momentum:

$$
\begin{aligned}
& \left(1 / N_{n}\right) d / d r\left(N_{n} T_{n}\right) \\
& \quad=1 / 2 m_{i} v_{i}^{2} N_{n}\left[2 m_{n} /\left(m_{i}+m_{n}\right)\right] \sigma_{i n}\left(n_{i} / N_{n}\right) .
\end{aligned}
$$

The electron energy balance equation:

$$
\begin{aligned}
\left(1 / r^{2}\right) d / d r\left(r^{2} q_{e}\right)= & (1 / \sigma) e^{2} z_{r}^{2} n_{i}^{2} v_{i}^{2}-\left(T_{e}-T_{n}\right) n_{i} \nu_{\mathrm{eq}} \\
& -3 / 2 n_{e} v_{e} d T_{e} / d r+T_{e} v_{e} d n_{e} / d r,
\end{aligned}
$$

where $q_{e}=-\kappa_{e} d T_{e} / d r$ is the electron thermal flux $\left(\kappa_{e}\right.$ is the electron thermal conductivity), $\nu_{\text {eq }}$ $=(3 / 2) \delta_{e n} v_{\text {the }} \sigma_{e n} N_{n}$ is the energy equipartition frequency between electrons and neutrals (the corresponding one between electrons and ions is negligible), $\delta_{e n}$ is a nondimensional parameter which measures the electron-neutral energy transfer per collision $\left(\delta_{e n} \sim 10^{-5}\right.$ for monatomic gases, while $\delta_{e n} \sim 10^{-3}$ for diatomic gases ${ }^{22}$ ), $v_{\text {the }}$ is the electron thermal velocity, and $\sigma_{e n}$ is the cross section for elastic scattering between electrons and neutrals.

And, finally, the neutral energy balance equation:

$$
\begin{aligned}
\left(1 / r^{2}\right) d / d r\left(r^{2} q_{n}\right)= & \left(1 / 2 m_{i} v_{i}^{2}\right) n_{i} v_{i} N_{n} \sigma_{i n} \delta_{i n} \\
& +\left(T_{e}-T_{n}\right) n_{i} \nu_{\mathrm{eq}},
\end{aligned}
$$

where $q_{n}=-\kappa_{n} d T_{n} / d r$ is the neutral thermal flux ( $\kappa_{n}$ is the neutral thermal conductivity) and $\delta_{i n}$ is the fraction of energy transfer per ion-neutral collision, $\delta_{i n}=2 m_{i} m_{n} /\left(m_{i}\right.$ $\left.+m_{n}\right)^{2}$.

The solution of Eqs. (1)-(8) depends on several parameters and of boundary values for the physical quantities. The arc characteristic determines $I_{r}=4 \pi a^{2} z_{r}$ e $n_{i}(a) v_{i}(a), m_{i}$, $m_{n}, a, T_{e}(a)$, and $E_{i}(a)$, while $\varphi(a)$ is taken equal to $V_{A}$. Boundary values for $N_{n}(a), v_{e}(a)$, and, since Eqs. (7) and (8) are second degree, one additional boundary condition for $T_{e}$, and two for $T_{n}$ must also be provided.

$N_{n}(a)$ is determined so that the resulting density profile $N_{n}(r)$ satisfies the conservation of the gas particle number, that is,

$$
\int N_{n}(r) r^{2} d r=2.4 \times 10^{16} p\left(r_{w}^{3}-a^{3}\right),
$$

where $p$ is the value of the neutral gas filling pressure and $r_{w}$ is the radius of the discharge chamber. In Eq. (9), $p$ is expressed in mbar and the rest of the quantities are in cgs units. Condition (9) expresses the fact that no pumping is going on during the discharge and, moreover, the "gettering" effect of neutrals by the arc can be neglected because the filling neutral density is between two and three orders of magnitude higher than the ion density.

For the determination of $v_{e}(a)$, we have resorted to our previous work. ${ }^{15}$ According to it, electrons are dragged from the arc in order to compensate for the electron losses due to ion-electron recombination in the whole outer region. Since this recombination occurs probably with the mediation of a charge-exchange process with the gas, ${ }^{23}$ the fast code of Ref. 15 was run in each case to obtain a good estimation of the neutral-gas density and the corresponding ion-current attenuation (for a given value of $\sigma_{\text {loss }}$ ), which in turn determines $v_{e}(a)$.

As shown later, for most of the practical cases of interest for this work, the plasma potential in the vicinities of the chamber wall results higher than the wall (ground) potential. Hence, a positively charged layer builds up close to the wall, where the electrons are elastically reflected. Under this con- 
dition, the electron heat flux to the wall is zero, thus requiring $\left.d T_{e} / d r\right) r_{w}=0 .{ }^{16}$ The other conditions employed for the neutral temperature are $T_{n}\left(r_{w}\right)=T_{\text {amb }}$ (being $T_{\text {amb }}$ the ambient temperature) and $\left.d T_{n} / d r\right) a=0$. The last boundary condition means that in the steady state there is no net thermal power flux from the arc plasma to the neutrals, as a consequence of the very low collision rate among electrons, ions and neutrals in the arc channel.

As was quoted in the previous section, when the ion kinetic energy is small enough, the model equations present a critical point. To see this, consider that in the ion slowing down region $\left(r<r^{*}\right)$, and for not too high neutral gas pressure values ( $p<5 \mathrm{mbar}$ ), the ion loss term in Eq. (1), and the ion-electron friction force in Eqs. (4) and (5) are very small, so these equations can be simplified and combined to obtain

$$
\begin{aligned}
{[1-} & \left.\left(T_{n}+z_{r} T_{e}\right) /\left(2 E_{i}\right)\right] d E_{i} / d r \\
= & -d / d r\left(T_{n}+z_{r} T_{e}\right)+2\left(T_{n}+z_{r} T_{e}\right) / r \\
& -2 E_{i} N_{n}\left[m_{n} /\left(m_{i}+m_{n}\right)\right] \sigma_{i n} .
\end{aligned}
$$

It is clear from Eq. (10) that at the point $r$ where the ion energy reaches the value $\left(T_{n}+z_{r} T_{e}\right) / 2$ (the critical point $\left.r^{*}\right)$, the derivative of the ion energy is infinite unless the right-hand side of Eq. (10) vanishes appropriately at the same point. This in turn imposes a condition on the derivative of the combination $T_{n}+z_{r} T_{e}$ at the critical point. Moreover, by Taylor expanding all magnitudes in Eq. (10) around the critical point, the correct (finite) derivative of $E_{i}$ can be obtained at this point. In this way, one could in principle use these boundary conditions at $r=r^{*}$ to implement a shooting method between $r=a$ and $r=r^{*}$, coupled to a similar integration between $r=r^{*}$ and $r=r_{w}$. However, there are three major difficulties with this approach (i) the position of $r^{*}$ is not known a priori (ii) the different derivatives at $r^{*}$ are very complex, and (iii) there is a large number of conditions to be adjusted at each boundary. Although these problems can certainly be solved, it turns out to be much more convenient to solve a pseudo time-dependent version of Eqs. (1)(8) and, starting with an initial guess, let the system relax to the stationary state. The time-dependent system does not present a critical point; all time derivatives are well defined at every point $r$. Since the time dependence is used for relaxation purposes only, we found it convenient to include this dependence only in Eqs. (1), (4), (7), and (8), adding to the left-hand sides the terms $\partial n_{i} / \partial t, \quad m_{i} \partial v_{i} / \partial t$, $3 / 2 \partial\left(n_{e} T_{e}\right) / \partial t$, and $3 / 2 \partial\left(N_{n} T_{n}\right) / \partial t$, respectively. Once these equations are evolved a time step, Eq. (2) is employed as it stands [together with Eq. (3)], to obtain the evolved profile of $v_{e}$. In the same way, Eq. (6) is used as it stands to obtain the evolved profile of $N_{n}$. We found this approach to be extremely efficient by obtaining very fast convergence to the same final state from very different "initial" conditions. The numerical procedure used is an explicit, predictor corrector, second-order accuracy in time scheme, with centered spatial differences.

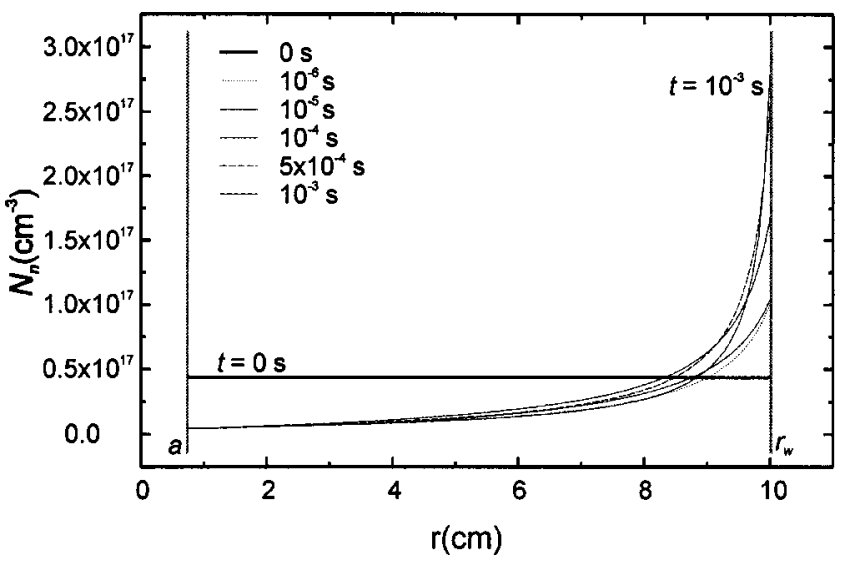

FIG. 2. Time evolution of the neutral gas density, according to the relaxation method employed to solve the equations. The "initial" gas density corresponds to the filling pressure value.

\section{RESULTS}

In what follows, we will present the results of the model for an arc operating in $\mathrm{Ar}$ gas at a filling pressure $p$ $=1.8$ mbar with a copper cathode $\left[z_{r}=1.85\right.$ (Ref. 18)]. The values of the other discharge parameters are: $I_{r}=10 \mathrm{~A}, a$ $=0.75 \mathrm{~cm}, \quad T_{e}(a)=2 \mathrm{eV}, \quad \varphi(a)=15 \mathrm{~V}, \quad E_{i}(a)=51.8 \mathrm{eV}$, $n_{i}(a)=3.8 \times 10^{12} \mathrm{~cm}^{-3}$ [obtained from Eq. (1)], and $r_{w}$ $=10 \mathrm{~cm}$. The $E_{i}(a)$ value corresponds to the average value of the kinetic energy of copper ions for an arc with $I$ $\sim 100 \mathrm{~A}$ of discharge current, experimentally determined by Davis and Miller ${ }^{24}$ once the corrections of the plasma potential of the $\operatorname{arc}^{4}$ have been performed. The $T_{e}(a)$ value was selected following previous experimental results ${ }^{25}$ for an arc with similar characteristics to those appropriate for the application of this model. A numerical solution of the model of Ref. 15 for the above quoted set of parameters, and with $\sigma_{\text {loss }}=2 \times 10^{-17} \mathrm{~cm}^{-2},{ }^{23}$ showed that in this case the ion current losses were almost total, thus giving $v_{e}(a)=v_{i}(a)$.

Since in the relaxation numerical method, one starts from arbitrary initial conditions, the first point to verify is the presence of a steady state in the solution. In Fig. 2, the temporal evolution of the neutral gas density is shown, starting from a constant density value (equal to that corresponding to the filling pressure value). It can be seen that for $t \sim 1 \mathrm{~ms}$, a steady state is reached, notwithstanding the fact that the original state of the neutral gas was very far from the final solution. However, this evolution does not represent the true transient stage of the problem, since in the equations of the model the neutral gas is assumed to be at rest.

In Fig. 3, the profiles of $n_{i}$ and $T_{e}$ [Fig. 3(a)], $v_{i}$ and $\varphi$ [Fig. 3(b)] and $N_{n}$ and $T_{n}$ [Fig. 3(c)] are presented. For comparison purposes, the profiles of the same quantities given by the model of Ref. 15 (valid only for $r<r^{*}$ ) are also presented for all cases. It can be seen from Figs. 3 that the profiles obtained from the model are very similar to those from Ref. 15 (for $r<r^{*}$ ), with the exception of $N_{n}$ and $T_{e}$. In the present case, $T_{e}$ is almost uniform, with a value equal to its value at the arc channel position. The difference in the $N_{n}$ profile is a consequence of the replacement of the bound- 

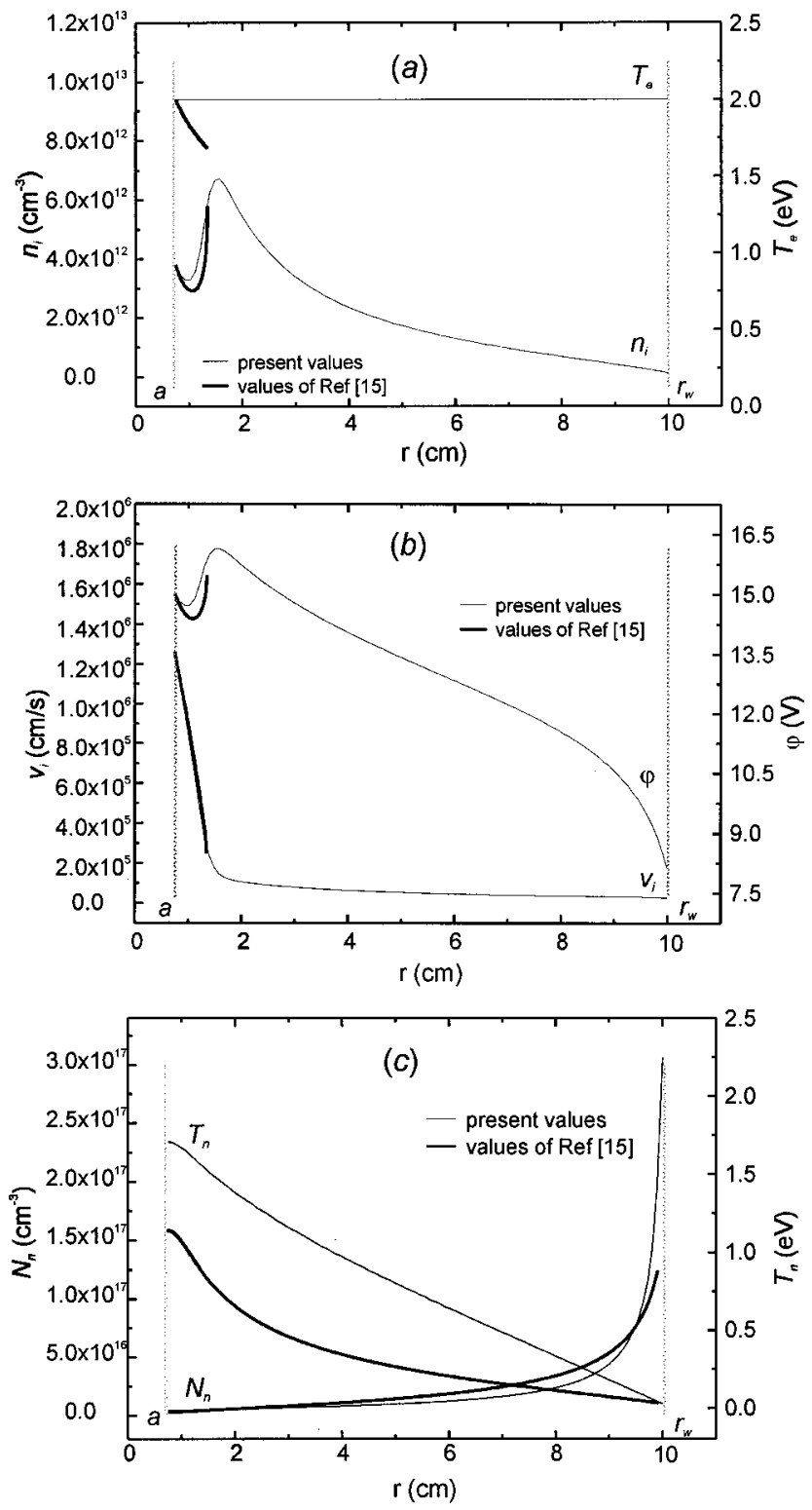

FIG. 3. Profiles of several physical quantities of the plasma-neutral gas structure, for an arc operating in $\mathrm{Ar}$ gas at a filling pressure $p=1.8 \mathrm{mbar}$ with a copper cathode $\left(z_{r}=1.85\right)$. The values of the other discharge parameters are: $I_{r}=10 \mathrm{~A}, \quad a=0.75 \mathrm{~cm}, \quad T_{e}(a)=2 \mathrm{eV}, \quad \varphi(a)=15 \mathrm{~V}, \quad E_{i}(a)$ $=51.8 \mathrm{eV}, r_{w}=10 \mathrm{~cm}$ and with $\sigma_{\mathrm{abs}}=2 \times 10^{-17} \mathrm{~cm}^{-2} \cdot n_{i}$ and $T_{e} ; v_{i}$ and $\varphi$; $N_{n}$ and $T_{n}$. The thick lines, extending between $r=a$ and the critical point $r=r^{*}$, correspond to the result of the model of Ref. 15 .

ary condition used in Ref. 15 (gas pressure at the wall equal to the filling pressure) by Eq. (9).

The peculiar behavior of the $n_{i}$ profile can be understood in terms of Eq. (1). For small $r$ values, the neutral density is small enough so as to neglect the metallic-ion losses, hence Eq. (1) can be written approximately as $r^{2} n_{i}(r) v_{i}(r)$ $\approx$ const. Since for small $r$ values, the ion slowing down is not significant yet, $n_{i}$ decreases with $r$, following the approximate relationship $n_{i} \sim r^{-2}$. For larger $r$ values $\left(r \rightarrow r^{*}\right)$, the ion slowing down is appreciable, giving place to an increase of $n_{i}$. This process does not continue indefinitely, because once the ion velocity becomes smaller than $v_{i}^{*}\left(v_{i}^{*}=2.8\right.$ $\times 10^{5} \mathrm{~cm} / \mathrm{s}$ at $r^{*}=1.36 \mathrm{~cm}$ for this example), it presents a very gentle decrease with $r$ [because the electrostatic force almost balances the neutral drag force for $r \geqslant 1.5 \mathrm{~cm}$, see Fig. 3(b)], so $n_{i}$ decreases with $r$ in this region due basically to geometrical effects and ion losses. As a result of the above quoted competing effects, the ion density reaches a maximum value in the vicinities of $r^{*}$ which almost doubles its value at the edge of the arc channel, while at the chamber wall position, its value is only $3 \%$ of $n_{i}(a)$ as a consequence of the strong ion loss for large $r$ values, where the neutral density is very high [see Fig. 3(c)].

Figure 3(b) shows that the $\varphi$ profile follows approximately the behavior of $n_{i}$, because in Eq. (5) the ionelectron drag and the electron-neutral momentum transfer are much smaller than the electron pressure and the electrostatic force, so these last two terms almost balance. For $r>r^{*}, \varphi$ is always a decreasing function of $r$, thus providing the proper force to the ions to overcome the neutral drag and reach the chamber wall. Note that the total voltage drop in the quasineutral structure $\left(a<r<r_{w}\right)$ is around $7 \mathrm{~V}$, so the last $8 \mathrm{~V}$ must drop inside the positive sheath adjacent to the wall. The neutral density continuously increases [starting from an initial value of $4.5 \times 10^{15} \mathrm{~cm}^{-3}$, that is $\approx 10 \%$ of the original neutral density, see Fig. 3(c)] as a consequence of a decrease in its temperature (because of neutral heat conduction) and an increase in the neutral pressure due to the impulse transferred by the ions. It reaches at the wall a value almost an order of magnitude higher than that corresponding to the filling pressure value. The gas temperature is high close to the $\operatorname{arc}\left[T_{n}(a)=1.7 \mathrm{eV}\right]$, and decreases monotonically up to the ambient temperature at the wall position.

In Fig. 4, the same profiles as those presented in Fig. 3 are shown. They correspond to the same arc parameters, but for $p=0.08$ mbar. From Fig. 4(a), it can be seen that in this case, the $n_{i}$ profile does not present the peculiarities showed in the previous high pressure case. Since now $r^{*}=6.5 \mathrm{~cm}$, the geometrical factor $r^{-2}$ in Eq. (1) prevails over the ion slowing down effect, and $n_{i}$ decreases monotonically in the major part of the chamber, presenting only a slight increase after the critical point. Starting from a value of 3.8 $\times 10^{12} \mathrm{~cm}^{-3}$ at $r=a$, it reaches near the wall a value of $\approx 30 \%$ of the density at the arc channel. This behavior of $n_{i}$ produces a corresponding spatial evolution of $\varphi$, which also decreases monotonically with $r$ up to the critical point. The total voltage drop in the plasma region reach $\approx 4.5 \mathrm{~V}$, so the remaining $10.5 \mathrm{~V}$ must fall in the positive sheath.

\section{DISCUSSION}

The solution derived from Eqs. (1) to (9) is in general similar to that presented in Ref. 15 for $r<r^{*}$. Hence, the characteristic features of the structure such as the penetration of neutrals into the metallic plasma, the heating and rarefaction of the neutral gas in the region where the ion kinetic energy is delivered, and the existence of an electrostatic voltage profile which roughly follows the ion density behavior are corroborated by the new solution. However, the numerical scheme has allowed to overcome the problem of the critical point and to obtain a solution valid up to the chamber wall position, with the only limitation of assuming a positively charged boundary layer in the vicinities of the cham- 

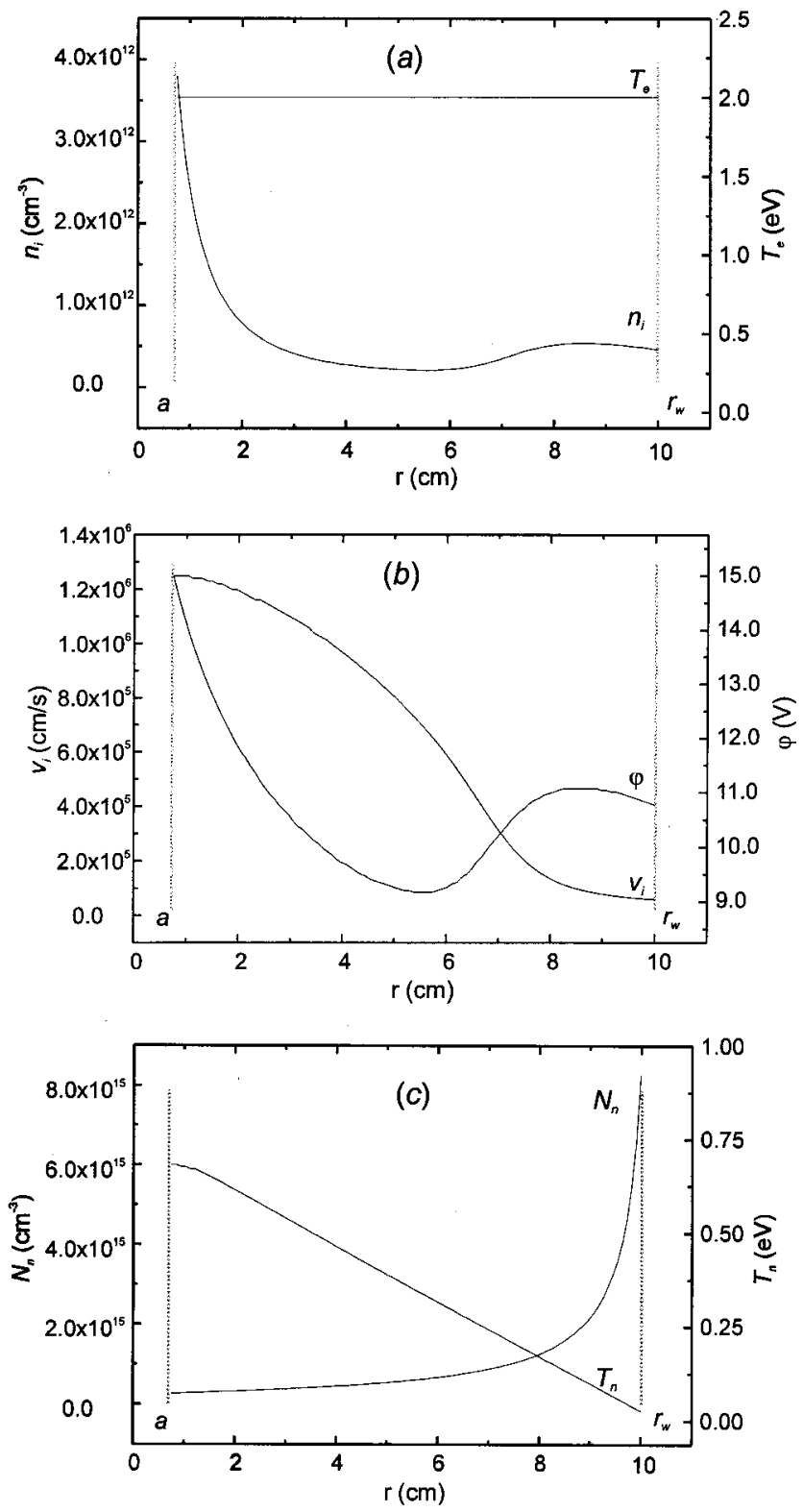

FIG. 4. Same profiles as those presented in Fig. 3. They correspond to the same arc parameters, but for $p=0.08$ mbar.

ber wall. The function of this layer is merely the matching of the electrostatic potential from its value in the plasma adjacent to the wall $\left[\varphi\left(r_{w}\right)\right]$ down to the ground wall potential. With the exception of very large discharge chambers and very low gas pressure values, it is found that $\varphi\left(r_{w}\right)>0$ (that is, a positive layer must exists), a fact that explains why in experiments in which current collectors biased to ground potential are used, they always collect positive (ion) charges. ${ }^{6,26}$

A fundamental point concerns the validity of the solution obtained. In this respect, it is conceivable that the smooth, regular-at-the-critical-point solution may be unstable, thus not realizable in practice. To settle this point, an exhaustive stability analysis should be performed, which is certainly very complex and beyond the scope of this work. However, we believe that the following points are indicative of a stable solution: (1) in Ref. 15, the steady-state solution up to the critical point was obtained directly from the steady-state equations using a high-accuracy Runge-Kutta integration scheme, and no other kind of solutions were found than those to which the relaxational scheme used in the present work converges. (2) Althought the system of equations solved is not the real time-dependent system, the time dependency was kept in equations likely to contribute to the development of instabilities. However, we have checked that the same final solution is obtained (for given values of the parameters of the problem) starting with very different "initial" guesses.

An important difference arising between the old solution ${ }^{15}$ and that presented in this work concerns the electron temperature behavior, a fact related to the election of the boundary conditions for $T_{e}$. In the old solution, the radial derivative of $T_{e}$ at the arc channel border was taken as the same value corresponding to a vacuum arc (without gas filling). This was done because of the practical difficulties in reaching the wall position with a shooting-type method. However, a simple calculation of the total power delivered by the thermal electron flux from the arc channel to the outer region shows that, for typical $\left.d T_{e} / d r\right)_{a}$ values $(-1--2 \mathrm{eV} /$ $\mathrm{cm}$ ), this flux results too high (of the order of several hundred of W) to be reliable. In the present case, the use of the more meaningful condition $\left.d T_{e} / d r\right) r_{w}=0$ leads to an almost constant electron temperature profile, thus indicating that the electron thermal flux from the arc to the outer region is very small. This seems to be an acceptable condition, since during the steady state of the arc the power delivered by the external circuit into the positive column of the arc plasma (joule heating) must be small.

The ion density profile obtained in this work presents a complicate shape which reflects the competence between two effects: the spherical expansion of the plasma, which produces a decreasing density profile, and the ion slowing down, which according to the ion continuity equation, tends to increase the density. For low pressures and large chambers, the geometrical factor dominates in the ion continuity equation, so the resulting ion density profile monotonically decreases according to the law $n_{i} \alpha r^{-2}$ (without ion losses); for high pressures and small chambers, the ion density profile takes a complicated shape, with a first minimum produced by the geometrical effects, followed by a maximum (sometimes higher than the ion density at the arc channel) produced in the vicinities of $r^{*}$.

The use of the conservation of the gas particle number [Eq. (9)] instead of the old condition requiring the equality between the neutral gas pressure (for $r>r^{*}$ ) and the prescribed value of the filling pressure, does not produce qualitative changes in the general plasma-neutral gas structure, but increases the density of the neutral particles close to the arc. In practice, with the use of the old condition, the neutral densities in the vicinities of the arc amounted to typically $1 \%$ of the neutral filling densities, while with the use of Eq. (9) the same fraction is about $10 \%$. Of course, this implies a change in the value of $r^{*}$, which now is smaller than that obtained in Ref. 15. In any case, the present $r^{*}$ values are still larger than those obtained from a balance between the plasma jet momentum flux and the background pressure. ${ }^{14}$ This balance is based on the existence of a shock wave sepa- 
rating the plasma and the gas, whose presence is not confirmed by any of our steady calculations, we then think that the balance of Ref. 14 is appropriate during a short initial transient of the discharge (when the neutral gas is not strongly disturbed yet and the mean free path for the ion energy deposition is small), but as the ion energy deposition heats and rarifies the gas, the mean free path becomes larger and the shock weaker, thus producing an interpenetration of the gas into the plasma until the final steady structure is reached. It is then expected that the steady values of $r^{*}$ should be larger than those calculated on the basis of a shock wave.

\section{FINAL REMARKS}

The model presented to describe the interaction between the metallic plasma and the neutral gas permits to obtain a complete steady state structure attained in the outer region of a low-pressure vacuum arc. The obtained solution represents a considerable improvement on that obtained previously employing a different numerical scheme ${ }^{15}$ for the integration of the equations. Since the plasma-neutral-gas structure we are dealing with is very complex, the availability of theoretical profiles of several physical quantities is useful for the interpretation of experimental results. For instance, the interpretation of Langmuir probe measurements strongly rests on the neutral gas state, because the collision mean free path of the charged particles is usually dictated by collisions with neutral particles. The neutral gas state in this kind of arcs has never been determined, so a theoretical prediction is necessary in order to assert the collisional (or noncollisional) nature of a probe boundary layer. In addition, it is known that the obtention of the plasma potential from the probe characteristic in low-pressure arcs is a complicated problem (because of either the uncertainty of the measurements due to the presence of high-frequency spikes or the deviations from the expected probe curve which arise at high electron currents), ${ }^{25,27}$ so the knowledge of the theoretical-value of the potential gives a good reference value to confront with experimental results. Also, in experiments where the ion attenuation is determined, ${ }^{6-9}$ the knowledge of a theoretical plasma density profile is useful in order to derive effective loss cross sections.

Another interesting feature of the obtained physical profiles is the determination of a base structure to study the excitation, deexcitation, ionization, and recombination of the neutral gas, which is a necessary step for the interpretation of spectroscopic measurements in the arc. Efforts on these improvements are currently under way.
In conclusion, the availability of a theoretical model for the outer region of this type of arcs constitutes a valuable tool for guiding experimental research and also to obtain an insight on the influence of several arc parameters, which are not easily accessible to experimental study.

\section{ACKNOWLEDGMENTS}

This work was supported by grants from the Buenos Aires University and CONICET.

${ }^{1}$ R. L. Boxman and S Goldsmith, IEEE Trans. Plasma Sci. PS-17, 705 (1989).

${ }^{2}$ P. A. Linfords, W. M. Mularie, and G. K. Wehner, Surf. Coat. Technol. 29, 275 (1986).

${ }^{3}$ C. W. Kimblin, J. Appl. Phys. 44, 3074 (1973).

${ }^{4}$ J. Kutzner and H. C. Miller, IEEE Trans. Plasma Sci. PS-17, 688 (1989).

${ }^{5}$ C. W. Kimblin, J. Appl. Phys. 45, 5235 (1974).

${ }^{6}$ H. Randhawa, Thin Solid Films 167, 175 (1988).

${ }^{7}$ S. Anders and B. Juttner, IEEE Trans. Plasma Sci. PS-19, 705 (1991).

${ }^{8}$ M. M. M. Bilek, P. J. Martin, and D. R. Mckenzie, J. Appl. Phys. 83, 2965 (1998).

${ }^{9}$ D. Grondona, H. Kelly, and A. Márquez, Astrophys. Space Sci. 256, 511 (1997).

${ }^{10}$ R. L. Boxman, S. Goldsmith, S. Shalev, H. Yaloz, and N. Brosh, Thin Solid Films 139, 41 (1986).

${ }^{11}$ H. Bruzzone, H. Kelly, A. Márquez, D. Lamas, A. Ansaldi, and C. Oviedo, Plasma Sources Sci. Technol. 5, 582 (1996).

${ }^{12}$ J. L. Meunier and M. G. Drouet, IEEE Trans. Plasma Sci. PS-15, 515 (1987).

${ }^{13}$ J. L. Meunier, IEEE Trans. Plasma Sci. PS-18, 904 (1990).

${ }^{14}$ R. L. Boxman and S. Goldsmith, IEEE Trans. Plasma Sci. PS-18, 231 (1990).

${ }^{15}$ H. Kelly, A. Márquez, and F. Minotti, IEEE Trans. Plasma Sci. PS-26, 1322 (1998)

${ }^{16}$ H. Kelly, A. Márquez, F. Minotti, and C. Ferro Fontán, J. Phys. D: Appl. Phys. 31, 1737 (1998)

${ }^{17}$ S. Goldsmith, in Handbook of Vacuum Arc Science and Technology, edited by R. L. Boxman, P. J. Martin, and D. M. Sanders (Noyes, New Jersey, 1995), p. 283.

${ }^{18}$ J. Kutzner and H. Craig Miller, J. Phys. D: Appl. Phys. 25, 686 (1992).

${ }^{19}$ E. M. Epperlein and M. G. Haines, Phys. Fluids 29, 1029 (1985).

${ }^{20}$ V. E. Golant, A. P. Zilinskij, and S. E. Sacharov, in Fundamentals Plasma Physics (in Italian) (MIR, Moscow, 1983).

${ }^{21}$ D. L. Book, in Plasma Physics, Physics Vade Mecum, edited by H. L. Anderson (AIP, New York, 1981), Chap. 18.

${ }^{22}$ Y. P. Raizer, Gas Discharge Physics (Springer, Berlin, 1991).

${ }^{23}$ J. L. Meunier and M. Douyon de Acevedo, IEEE Trans. Plasma Sci. PS-20, 1053 (1992).

${ }^{24}$ W. D. Davis and H. C. Miller, J. Appl. Phys. 40, 2212 (1969).

${ }^{25}$ D. Grondona, H. Kelly, A. Márquez, and F. Minotti, J. Phys. D: Appl. Phys. 31, 3358 (1998)

${ }^{26}$ D. Grondona, H. Kelly, and A. Márquez, Astrophys. Space Sci. 256, 511 (1997).

${ }^{27}$ S. M. Shkolnik, IEEE Trans. Plasma Sci. PS-13, 336 (1985). 